\title{
Variables cognitivas e ingreso universitario: predictores del rendimiento académico*
}

\section{Cognitive Variables and University Entrance: Predictors of Academic Achievement}

Recibido: junio 6 de 2013 | Revisado: abril 18 de 2014 | Aceptado: abril 18 de 2014

\author{
María Antonieta Elvira-VAldÉS ** \\ LYDIA PUJOL *** \\ Universidad Simón Bolívar, Caracas, Venezuela
}

doi.org/10.11144/Javeriana.UPSY13-4.vciu

Para citar este artículo: Elvira-Valdés, M. A., \& Pujol, L. (2014). Variables cognitivas e ingreso universitario: predictores del rendimiento académico. Universitas Psychologica, 13(4), 1557-1567. http:// dx.doi.org/10.11144/Javeriana.UPSY13-4.vciu

* Investigación desarrollada bajo la línea de investigación Psicoeducativa adscrita al Dpto. de Ciencia y Tecnología del Comportamiento de la Universidad Simón Bolívar

** Profesora e investigadora. Departamento Ciencia y Tecnología del Comportamiento. Correo electrónico:melvira@usb.ve

***** Profesora e investigadora. Departamento Ciencia y Tecnología del Comportamiento. Correo electrónico:1pujol@usb.ve

\section{RESUMEN}

La presente investigación se planteó como objetivo determinar el poder predictivo de las fases de autorregulación académica, los estilos de aprendizaje y la modalidad de ingreso al sistema universitario, sobre el rendimiento académico de los estudiantes. La muestra estuvo constituida por 214 estudiantes voluntarios, cursantes del ciclo básico de carreras tecnológicas de la Universidad Simón Bolívar. En la medición de variables se empleó la Escala de Autorregulación Académica ([PCR]; Lezama, 2005) y el Cuestionario Honey-Alonso de Estilos de Aprendizaje ([CHAEA]; Alonso, Gallego, \& Honey, 1997). El procesamiento de datos se realizó mediante asociaciones bivariadas y análisis multivariados de regresión múltiple. Los hallazgos obtenidos evidenciaron que la autorregulación académica en su fase de control, la preferencia por el estilo de aprendizaje teórico y el ingreso a la universidad por prueba interna son variables predictoras del rendimiento académico. Palabras clave

autorregulación académica; estilos de aprendizaje; ingreso universitario; rendimiento académico; carreras tecnológicas

\section{A B S T R A C T}

This research was designed to determine the predictive power of academic self-regulation phases, learning styles and mode of entry into university system, on the academic achievement of students. The sample consisted of 214 volunteer students of basic cycle technology carrers at Simon Bolivar University. On measuring of variables was used PCR Academic Self-Regulation Scale (Lezama, 2005) and the Honey-Alonso CHAEA Learning Styles (Alonso, Gallego, \& Honey, 1997). The data processing was performed using bivariate associations and multiple regression multivariate analysis. The findings evidenced that academic self-regulation in control phase, preference for theorist learning style and university entrance by internal test, are significant predictors of academic achievement.

Keywords

Academic self-regulation; learning styles; university entrance; academic achievement; technology careers 


\section{Introducción}

Durante las últimas décadas, la teoría e investigación sobre la autorregulación académica se ha convertido en uno de los ejes principales para la acción educativa. Si bien las primeras investigaciones sobre la autorregulación se realizaron en contextos terapéuticos, dirigidos a la modificación de conductas disfuncionales (Schunk, 1998), consecuentemente la atención se fue ampliando hacia las fases y procesos que subyacen al aprendizaje autorregulado, ante la posibilidad de generar en los estudiantes el ser estratégicos y la posibilidad de autodirigir su motivación hacia metas valiosas y alcanzables (Carbonero \& Navarro, 2006; García-Ros \& PérezGonzález, 2011; Núñez, Solano, González-Pienda, \& Rosário, 2006; Pintrich, 2000; Zimmerman \& Schunk, 2001). Numerosas investigaciones se han desarrollado con el tema de la autorregulación, ofreciendo valiosas contribuciones en el medio académico (González-Pienda, Núñez, Álvarez, \& González, 2002; Greene, Moos, Acevedo, \& Winters, 2008; Malmberg, Järvenoja, \& Järvelä, 2010; Schunk, 2005; Valle, Canabach, Rodríguez, Núñez, \& González-Pienda, 2006; Virtanen \& Nevgi, 2010). Los estudios apuntan hacia un fenómeno complejo y multifacético.

La autorregulación académica o aprendizaje autorregulado como constructo, está relacionado con formas de aprendizaje independientes y efectivas (Montes, Ayala, \& Atencio, 2005; Núñez et al., 2006) y busca explicar cómo la persona aumenta sus resultados académicos usando un método de forma sistemática (Lamas, 2008; Suárez \& Fernández, 2004). Pintrich (2000) plantea que la autorregulación académica es:

(...) un proceso activo y constructivo mediante el cual los aprendices fijan metas para su aprendizaje e intentan monitorear, regular y controlar su cognición, motivación y conducta, guiados y restringidos por sus metas y las características contextuales de su ambiente. (p. 453)

El modelo propuesto por Pintrich (2000), sugiere que el desarrollo de la autorregulación se produce a través de áreas (cognición, motivación, conducta, contexto) y fases (planificación, control, reflexión). Comprendida como un proceso activo, cíclico y recurrente, las fases de la autorregulación no están estructuradas de manera jerárquica ni lineal, sino que se manifiestan de manera dinámica, produciéndose interacciones entre las mismas. Para cada fase, en función de las demandas que precise la tarea de aprendizaje, se esperan determinadas actividades más o menos estratégicas del estudiante que le permitan regular aspectos cognitivos, motivacionales y contextuales en la resolución de la tarea (Álvarez, 2009; Zimmerman, Kitsantas, \& Campillo, 2005).

Los procesos autorreguladores arrancan en la fase de planificación, previsión y activación, en la que se destacan actividades importantes como el establecimiento de metas que se desean alcanzar, la activación del conocimiento previo sobre la materia y del conocimiento metacognitivo, la activación de las creencias motivacionales y del área afectiva, la planificación de tiempo y esfuerzo que se debe emplear en las tareas y las percepciones respecto a estas últimas y al contexto. Conforme a los resultados de la fase anterior, el estudiante pone en marcha actividades de control que comprenden la selección y utilización de estrategias para controlar el pensamiento, la motivación y el afecto, así como las relacionadas con la regulación de tiempo y esfuerzo, y control de las diversas tareas académicas y clima de estudio. Finalmente, la fase de reflexión y reacción, abarca los juicios y evaluaciones que los estudiantes realizan acerca de su ejecución en las tareas, comparándolas con los objetivos previamente establecidos, las atribuciones que realizan sobre las causas de sus éxitos o fracasos, las reacciones que experimentan ante los resultados, la elección del futuro comportamiento y las evaluaciones generales sobre la tarea y el ambiente de estudio (Green et al., 2008; Lamas, 2008; Pintrich, 2000; Valle et al., 2006).

Por otro lado, el constructo estilos de aprendizaje también ha generado abundante literatura en los últimos años, ante la necesidad y el desafío de conocer el estilo de aprender de los estudiantes para un mejor aprovechamiento de todas sus potencialidades (Marín, 2002; Pujol, 2008; Hervás, 2008; 
Laugero, Balcaza, Salinas y Craveri, 2009; LópezAguado, 2011; Segura, 2011). Conceptualmente, los estilos de aprendizaje se han asumido como variables personales que explican las diferentes formas de percibir, interaccionar y responder a los ambientes de aprendizaje; no obstante, la amplitud y variedad de teorías han dispuesto un abanico de clasificaciones que precisan más unos aspectos que otros (actitudinales, afectivos, cognitivos, ambientales). En la definición de estilos de aprendizaje propuesta por Keefe (1982), estos son concebidos de manera holística como "los rasgos cognitivos, afectivos y fisiológicos que sirven como indicadores relativamente estables de cómo los aprendices perciben, interaccionan y responden a sus ambientes de aprendizaje" (p. 44). Se coincide con Alonso, Gallego y Honey (1997) y con numerosas investigaciones en el área (Cázares, 2009; Cerezo, Casanova, Torre, \& Carpio, 2011; Gutiérrez et al., 2011; Hervás, 2008; Pujol, 2008; Laugero et al., 2009; López \& Silva, 2009) donde los estilos de aprendizaje son una descripción de las actitudes y conductas que determinan una manera de aprender preferida por un individuo (Honey \& Mumford, 1986).

En la propuesta de Alonso et al. (1997), se consideran como estilos los siguientes (se incluyen algunas de sus principales características): activo (sujetos que se implican plenamente en nuevas experiencias, con mente abierta que emprenden con entusiasmo las tareas nuevas, se crecen ante los retos y se aburren con los plazos largos); reflexivo (reúnen datos y los analizan detenidamente antes de formular conclusión alguna, son prudentes y les gusta considerar todas las alternativas posibles); teórico (enfocan los problemas de forma vertical y por etapas lógicas, tienden a ser perfeccionistas e integrar los hechos en teorías coherentes) y pragmático (actúan de forma rápida y segura ante ideas y proyectos que atraen su atención, tienden a ser impacientes ante la teorización, pues prefieren la aplicación práctica).

En esta investigación, también se incluye la variable ingreso a la universidad por considerarse un componente significativo en el área académica. En Venezuela, el tema del ingreso al sistema universitario constituye un problema cuya solución entra- ña un reto difícil y complejo (Cendrós, Aular, \& Marcano, 2008; Fuenmayor, 2002; Linares, 2000; Parra, 2001; Pérez \& Díaz, 2006). Durante años, la realidad de la admisión de los estudiantes a la universidad se ha visto influenciada por diversos y complicados factores, cuya existencia se extiende más allá del sistema de educación superior en sí mismo e involucra causas sociales implicadas en la grave crisis que se vive en la sociedad venezolana (Centro Interuniversitario de Desarrollo, 2006; Fuenmayor, 2003; Parra, 2001). La apreciación de un sistema de acceso a la universidad como excluyente y discriminativo ha dado origen a decisiones gubernamentales que han transformado en los años recientes los mecanismos de ingreso universitario (Morles, Medina, \& Álvarez, 2003; Oficina de Planificación del Sector Universitario, 2007, 2010; Pérez \& Díaz, 2006). La eliminación de la Prueba de Aptitud Académica en el año 2007, además del impedimento expreso a partir del año 2009 en cuanto a la aplicación de las pruebas voluntarias de ingreso en las universidades nacionales, reflejan que se está viviendo un período de transición en el sistema de educación superior venezolano (Cendrós et al., 2008; Centro Interuniversitario de Desarrollo, 2006; Fuenmayor, 2002). Al respecto, en la Universidad Simón Bolívar, recurriendo a las facultades que le otorga su autonomía universitaria y que la capacita para dictar sus propias normas de organización, ha seguido implementando el mecanismo de acceso a través de la prueba interna, a pesar de vulnerar los preceptos vigentes (Oficina de Planificación del Sector Universitario, 2010).

La realidad revela que los estudiantes que ingresan a las universidades lo hacen con una escasa formación y con deficiencias de conocimiento en general (Bolívar, \& Rojas, 2008; Cardozo, 2008; Montero, Sepúlveda, \& Contreras, 2011; Rodríguez, Fita, \& Torrado, 2004; Universidad Simón Bolívar, 2007, 2010), además de obtener un pobre rendimiento académico, sobre todo durante el primer período de su carrera, hecho que ha generado múltiples investigaciones en el área (Blumen, Rivero, \& Guerrero, 2011; Craveri \& Anido, 2009; De la Fuente, Pichardo, Justicia, \& Berbén, 2008; De la Orden, Oliveros, Mafokozi, 
\& González, 2001; De Garay, 2003; De Miguel, 2001; Garbanzo, 2007; Gargallo, 2006; Gargallo, Suárez, \& Ferreras, 2007; Juárez, HernándezCastro, \& Escoto, 2011). Esta situación que se impone en cuanto a la deficiente preparación de los bachilleres, en general, aunada a la problemática explicada con respecto del acceso al sistema universitario, ha hecho que en la Universidad Simón Bolívar desde el año 2005 se esté implementando el Ciclo de Iniciación Universitaria (CIU), que es un curso de nivelación para dar oportunidades a los estudiantes que no alcanzan el puntaje mínimo en la prueba interna de ingreso. Con el paso del tiempo, la universidad se ha visto en la necesidad de incrementar el porcentaje de alumnos admitidos al CIU, dado el bajo nivel de preparación que presentan los aspirantes que se someten a la prueba interna (Universidad Simón Bolívar, 2007, 2010).

En vista de todo lo expuesto, el interés de esta investigación es conocer los estilos de aprendizaje de los estudiantes que inician sus estudios en las carreras tecnológicas de la Universidad Simón Bolívar, cómo pueden ser caracterizados de acuerdo a su autorregulación académica, cuál ha sido la modalidad por la que han logrado el ingreso al sistema universitario y si todos estos factores pueden ser considerados influyentes o predictores del rendimiento académico que alcanzan durante su primer curso de estudios superiores. Identificar la preferencia de estilos de aprendizaje y la autorregulación que despliega el aprendiz durante su gestión de estudio, que está ingresando en una carrera universitaria por una modalidad de acceso específica, resulta valioso debido a la importancia por lograr que los estudiantes sean aprendices autónomos y exitosos, que sean capaces de regular su propio proceso de aprendizaje, lo que necesariamente debe originarse en el conocimiento de sus particularidades en estos factores, entre otros. En ese sentido, el objetivo del presente estudio es determinar el poder predictivo de las fases de autorregulación, los estilos de aprendizaje y la modalidad de ingreso al sistema universitario sobre el rendimiento académico de los estudiantes de carreras tecnológicas de la Universidad Simón Bolívar.

\section{Método}

\section{Participantes}

La muestra de este estudio quedó constituida por un total de 214 estudiantes de ambos sexos, 121 mujeres y 93 hombres, con una edad promedio de 17.73 años, cursantes del primer trimestre del ciclo básico de carreras tecnológicas que fueron admitidos a la Universidad Simón Bolívar por cualquiera de la tres modalidades de ingreso (prueba interna, CIU o asignación de cupos) y que decidieron participar de manera voluntaria. La selección de la muestra se realizó de forma intencional y no probabilística (Hernández, Fernández, \& Baptista, 2004).

\section{Instrumentos}

Escala de Aprendizaje Autorregulado PCR (Lezama, 2005)

Es un instrumento de autorreporte dirigido a examinar patrones de autorregulación del aprendizaje en las fases de planificación, control y reflexión. Está conformado por 15 reactivos (5 por cada subescala), que se responden a través de una escala tipo Likert de 6 puntos, arrojando una calificación con puntuación inversa (a menor puntaje mayor autorregulación) que oscila entre 15 y 90 puntos. La evaluación de las propiedades psicométricas de la escala se realizó con estudiantes de la Universidad Simón Bolívar (Elvira-Valdés \& Pujol, 2012) y los valores de consistencia interna estimados a través del alfa de Cronbach presentaron un resultado de 0.761 para la totalidad de la escala y valores similares para las subescalas.

Cuestionario Honey-Alonso de Estilos de Aprendizaje CHAEA (Alonso et al., 1997). Instrumento de autorreporte que consta de 80 ítems estructurados en cuatro grupos de 20 reactivos, que corresponden a los estilos activo, reflexivo, teórico y pragmático. La adaptación del cuestionario fue realizada por Pujol (2008) y, en un análisis más reciente (DuránAponte \& Pujol, 2012), las pruebas de confiabilidad arrojaron un alfa de Cronbach de 0.789 para la totalidad del instrumento. 


\section{Procedimiento}

Habiéndose explicado el propósito del estudio y solicitando su participación voluntaria, la aplicación de los instrumentos se realizó durante el horario habitual de clase de los estudiantes, con una duración aproximada de 20 minutos. Una vez finalizado el trimestre, se solicitó en Control de Estudios el índice académico de cada uno de los participantes. Recopilada toda la información, se diseñó la base de datos y se procedió a los análisis estadísticos, utilizando el programa SPSS versión 18.0 (PASW Statistics 18).

\section{Resultados}

Los resultados señalan que la muestra de esta investigación, formada por 214 estudiantes de primer trimestre de ciclo básico, se caracteriza por tener edades comprendidas entre los 16 y 20 años, siendo 17.73 la edad promedio. También se distingue por un mayor predominio del sexo femenino (56.5\%) que del masculino (43.5\%) y mayor cantidad de estudiantes graduados en instituciones privadas (58.9\%) en relación con las públicas (41.1\%). La mayoría de los participantes (54.7\%) ingresaron a la Universidad Simón Bolívar, aprobando la prueba interna; el 24.3\% de ellos ingresó una vez cursado y aprobado el CIU y el resto (21\%) logró el ingreso por asignación de cupo.

Como puede observarse en la Tabla 1, los límites para las subescalas planificación y control van entre
5 y 27 puntos, con un puntaje promedio de 15.85 en planificación y 14.62 en control. Las asimetrías bajas y positivas $(0.091$ y 0.275$)$, las curtosis $(-0.722$ y 0.3), desviaciones estándar (5.101 y 4.112) y coeficientes de variación (32.18 y 28.13) indican que la muestra tiende a distribuciones poco heterogéneas y moderado uso de estrategias de planificación y control. Por otro lado, la subescala reflexión presenta límites que van entre 5 y 30 puntos, así como una media de 11.1. La asimetría alta y positiva (1.64), la curtosis (2.653), la desviación estándar (5.377) y el coeficiente de variación (48.44) presentan una muestra que tiende a una distribución heterogénea y cuyos puntajes sugieren un mayor uso de estrategias de reflexión autorregulatoria.

Para la interpretación de los puntajes del CHAEA se elaboró el baremo de explicación propuesto por Alonso et al. (1997), el cual se estructura en función de los resultados de todos los participantes, lo que no solo permite comparar cada sujeto con el grupo al cual pertenece, sino también ubicarlo según su estilo de aprendizaje. El criterio de ubicación de los estudiantes que puntuaron igual en más de un estilo, fue ubicarlos en el nivel de preferencia en que la puntuación fuese alta o muy alta.

Como puede observarse en la Tabla 2, de la muestra del estudio 61 participantes (28.5\%) se ubican en el estilo reflexivo, lo que representa la mayoría de ellos. Esta cantidad viene seguida de 56 sujetos (26.2\%) que se ubican en el estilo pragmático, 52 sujetos (24.3\%) en el teórico y la menor

TABLA 1

Resultados estadísticos de las dimensiones de autorregulación académica

\begin{tabular}{|c|c|c|c|}
\hline Estadísticos & Planificación & Control & Reflexión \\
\hline Mínimo & 5 & 5 & 5 \\
\hline Máximo & 27 & 28 & 30 \\
\hline Media & 15.85 & 14.62 & 11.1 \\
\hline Mediana & 16 & 14 & 10 \\
\hline Desviación estándar & 5.101 & 4.112 & 5.377 \\
\hline Asimetría & 0.091 & 0.275 & 1.64 \\
\hline Curtosis & -0.722 & 0.3 & 2.653 \\
\hline Coeficiente de Variación & 32.18 & 28.13 & 48.44 \\
\hline
\end{tabular}

Fuente: elaboración propia 
TABLA 2

Distribución de los participantes según el estilo de aprendizaje preferido

\begin{tabular}{ccc}
\hline Estilo de Aprendizaje & Frecuencia & Porcentaje (\%) \\
\hline Activo & 45 & 21 \\
Reflexivo & 61 & 28.5 \\
Teórico & 52 & 24.3 \\
Pragmático & 56 & 26.2 \\
\hline & 214 & 100 \\
\hline
\end{tabular}

Fuente: elaboración propia

cantidad de ellos, 45 sujetos (21\%) se ubican en el estilo activo de aprendizaje.

Para el rendimiento, se utilizó el índice académico que se obtiene al término del trimestre, calculado multiplicando la nota de cada asignatura por las unidades de crédito correspondientes y que se expresa en una escala decimal que va de 1 a 5 puntos. El plan de estudios del primer trimestre del ciclo básico contempla las siguientes 4 asignaturas: Matemática I, Lenguaje I, Inglés I y Hombre, Cultura y Sociedad. El índice académico de la muestra presentó una media de 3.34 puntos, lo que representa un rendimiento promedio.

Los resultados de las correlaciones bivariadas mostradas en la Tabla 3 evidencian correlaciones moderadas y positivas entre los estilos de aprendizaje activo y pragmático $(r=0.501 ; p<0.001)$, reflexivo y teórico $(r=0.516 ; p<0.001)$, lo que sugiere que un mayor puntaje en el estilo activo está asociado de forma moderada con mayores puntajes en el pragmático; del mismo modo, mayor puntaje en el estilo reflexivo está asociado con mayor puntaje en el teórico. Asimismo, se presentan correlaciones bajas y positivas entre los estilos pragmático y reflexivo $(r=0.269 ; p<0.001)$, pragmático y teórico $(r=0.293 ; p<0.001)$, lo que sugiere que un mayor puntaje en el estilo pragmático está asociado de forma baja con mayores puntajes en los estilos reflexivo y teórico.

Respecto a las subescalas de autorregulación académica, se observa una correlación moderada y positiva entre control y reflexión $(r=0.459$; $p<$ $0.001)$, así como una correlación baja y negativa entre control y el estilo de aprendizaje activo $(r=$ $-0.215 ; p<0.001$ ), lo que sugiere que mayores puntajes en control están asociados de forma moderada

TABLA 3

Correlaciones de Pearson entre las variables

\begin{tabular}{|c|c|c|c|c|c|c|c|c|c|c|c|}
\hline Variables & 1 & 2 & 3 & 4 & 5 & 6 & 7 & 8 & 9 & 10 & 11 \\
\hline 1. Planificación & 1 & & & & & & & & & & \\
\hline 2. Control & $0.181^{* *}$ & 1 & & & & & & & & & \\
\hline 3. Reflexión & $0.283^{* *}$ & 0.459 & 1 & & & & & & & & \\
\hline 4. E-Activo & 0.046 & $-0.215^{* *}$ & -0.106 & 1 & & & & & & & \\
\hline 5. E-Reflexivo & $-0.259 * *$ & -0.003 & -0.069 & 0.057 & 1 & & & & & & \\
\hline 6. E-Teórico & $-0.212 * *$ & 0.019 & -0.064 & -0.007 & $0.516 * *$ & 1 & & & & & \\
\hline 7. E-Pragmático & -0.072 & -0.126 & -0.087 & $0.501 * *$ & $0.269 * *$ & $0.293 * *$ & 1 & & & & \\
\hline 8. Prueba int & 0.028 & -0.031 & $-0.164 *$ & -0.024 & $0.150 *$ & 0.015 & -0.002 & 1 & & & \\
\hline 9. Asignar cupo & -0.027 & -0.008 & 0.038 & -0.006 & -0.112 & -0.096 & -0.099 & $-0.167 *$ & 1 & & \\
\hline 10. CIU & -0.006 & 0.044 & $0.154 *$ & 0.034 & -0.068 & 0.073 & 0.096 & $-0.122 *$ & $-0.162 *$ & 1 & \\
\hline 11. R-Acad & -0.065 & $-0.139 *$ & -0.063 & -0.013 & 0.001 & 0.132 & 0.016 & $0.265^{* *}$ & $-0.260 * *$ & -0.061 & 1 \\
\hline
\end{tabular}

**Significativa al 0.01

*Significativa al 0.05

Fuente: elaboración propia 
con un mayor puntaje en la subescala reflexión y menor puntaje en el estilo activo. Por otra parte, planificación correlaciona de forma baja y positiva con las subescalas control $(r=0.181 ; p<0.001)$ y reflexión $(r=0.283 ; p<0.001)$ y de forma baja y negativa con los estilos de aprendizaje reflexivo $(r=-0.259 ; p<0.001)$ y teórico $(r=-0.212 ; p<$ 0.001 ); esto sugiere que mayores puntajes en planificación autorregulatoria están asociados de forma baja con mayor puntaje en las subescalas control y reflexión, así como menor puntaje en los estilos reflexivo y teórico.

Respecto al rendimiento académico, este correlaciona de forma baja y positiva con la modalidad de ingreso por prueba interna $(r=0.265 ; p<0.001)$, lo que sugiere que un mayor índice académico está asociado de forma baja con ingresar a la universidad por esta vía; por otro lado, el rendimiento también correlaciona con el ingreso por asignación de cupo, pero de forma baja y negativa $(r=-0.26 ; p<0.001)$, lo que sugiere que un menor índice académico está asociado con el ingreso por asignación. Por último, la Tabla 3 también da evidencia de algunas asociaciones bajas al 95\% de confianza, mientras que el resto de las variables presentó correlaciones que no resultaron significativas desde el punto de vista estadístico.

A fin de establecer el poder predictivo de la autorregulación académica, los estilos de aprendizaje y la modalidad de ingreso al sistema universitario sobre el rendimiento académico, se realizó una regresión múltiple con el método Entre, introduciendo todas las variables en el análisis. Para efectos de este estudio y que fuese aceptable para la regresión, la modalidad de ingreso fue recodificada por ser una variable politómica con más de dos categorías; en consecuencia, tomó valores codificados como 1 o 0 y se asumió como variable ficticia con $\mathrm{k}$-1 categorías o dummy (Hair, Anderson, Tatham, \& Black, 2000; Kerlinger \& Lee, 2002). Al transformar los datos, la modalidad de ingreso quedó convertida en dos variables binarias.

Una vez analizado el cumplimiento de los supuestos básicos que deben cumplirse (normalidad, linealidad, homocedasticidad e independencia de los términos de error) según Hair et al. (2000), en el modelo de regresión múltiple se obtuvo una correlación moderada $(R=461)$ entre las variables predictoras y el rendimiento académico. Este conjunto de variables logra explicar el $21.2 \%\left(R^{2}\right.$ $=0.212$ ) de la varianza total del rendimiento académico, con un error de estimación de 0.563. El contraste Durbin-Watson arrojó un valor de 2.329 lo que indica que los errores no están correlacionados entre sí, mientras que la prueba de análisis de varianza mostró la existencia de relación lineal entre el conjunto de variables predictoras y el rendimiento académico $(F=4,147 ; g l=13 / 200 ; p<$

TABLA 4

Coeficientes de regresión parcial

\begin{tabular}{|c|c|c|c|c|c|c|c|}
\hline \multirow[t]{2}{*}{ Modelo } & \multicolumn{2}{|c|}{$\begin{array}{l}\text { Coeficientes no } \\
\text { estandarizados }\end{array}$} & \multirow{2}{*}{$\begin{array}{c}\begin{array}{c}\text { Coeficientes } \\
\text { estandarizados }\end{array} \\
\text { Beta }\end{array}$} & \multirow[t]{2}{*}{$\mathrm{t}$} & \multirow[t]{2}{*}{ Sig. } & \multicolumn{2}{|c|}{$\begin{array}{l}\text { Estadísticos de } \\
\text { Colinealidad }\end{array}$} \\
\hline & $\mathrm{B}$ & Error típ. & & & & Tolerancia & FIV \\
\hline Constante & 2.87 & 0.469 & & 6.122 & 0 & & \\
\hline Planificación & -0.013 & 0.008 & -0.106 & -1.519 & 0.13 & 0.808 & 1.237 \\
\hline Control & -0.025 & 0.011 & -0.166 & -2.284 & 0.023 & 0.744 & 1.343 \\
\hline Reflexión & 0.01 & 0.009 & 0.084 & 1.124 & 0.262 & 0.713 & 1.403 \\
\hline E-Activo & -0.005 & 0.016 & -0.024 & -0.307 & 0.759 & 0.673 & 1.486 \\
\hline E-Reflexivo & -0.027 & 0.019 & -0.112 & -1.429 & 0.154 & 0.639 & 1.566 \\
\hline E-Teórico & 0.042 & 0.016 & 0.199 & 2.592 & 0.01 & 0.67 & 1.492 \\
\hline E-Pragmático & -0.006 & 0.018 & -0.024 & -0.311 & 0.756 & 0.638 & 1.566 \\
\hline Ingreso 1 & 0.339 & 0.106 & 0.225 & 3.188 & 0.002 & 0.791 & 1.265 \\
\hline Ingreso 2 & 0.307 & 0.092 & 0.168 & 1.751 & 0.081 & 0.699 & 1.43 \\
\hline
\end{tabular}

Fuente: elaboración propia 
0.001), con una significancia desde el punto de vista estadístico al 99\% de confianza.

En la Tabla 4 se aprecia que del conjunto de variables introducidas en el modelo de regresión aparecen relevantes para la ecuación de regresión: la subescala control de autorregulación académica, el estilo de aprendizaje teórico y la modalidad de ingreso 1. Asimismo, se observa la ausencia de multicolinealidad en las columnas correspondientes a tolerancia y sus inversos (FIV). Entre la subescala control autorregulatorio y el rendimiento académico se obtuvo una correlación baja y negativa ( $\beta$ $=-0.166 ; p=0.023$ ), significativa al $95 \%$ de confianza. Con el rendimiento, el estilo de aprendizaje teórico correlacionó de forma baja y positiva ( $\beta=$ $0.199 ; p=0.01$ ), mientras que la modalidad de ingreso 1 presenta una correlación baja y positiva $(\beta$ $=0.225 ; p=0.002$ ), ambas significativas al $99 \%$ de confianza. El resto de las variables introducidas en el análisis de regresión no resultaron significativas desde el punto de vista estadístico.

\section{Discusión}

La presente investigación tuvo como principal objetivo determinar el poder predictivo de las fases de autorregulación académica, los estilos de aprendizaje y la modalidad de ingreso al sistema universitario sobre el rendimiento académico de los estudiantes de carreras tecnológicas de la Universidad Simón Bolívar. En primer lugar, se encontró que los estudiantes mostraron un nivel alto en la subescala reflexión autorregulatoria y niveles moderados en las subescalas planificación y control. Esto sugiere que el estudiante, durante el inicio de su experiencia universitaria, despliega mayor uso de acciones para evaluar el contexto y la conducta académica que actividades para establecer sus metas, planificar tiempo, esfuerzo y contexto, además de seleccionar y utilizar estrategias de aprendizaje y monitoreo de su aplicación. Sin embargo, lo anterior no supone que el estudiante reaccione adoptando las tácticas más adecuadas para mejorar su desempeño, luego de valorar y reformular la planificación y ejecución de sus actividades.

Respecto a los estilos de aprendizaje, la mayoría de los estudiantes manifestó preferencia por el estilo reflexivo, seguido por los estilos pragmático, teórico y activo. Como gran parte de los resultados encontrados en la literatura, pareciera presentarse una mayor predominancia por el estilo reflexivo en el estudiantado, en general. Esto pudiera apuntar hacia una primacía por este tipo de estilo de aprendizaje, en un sistema educativo que privilegia las características de los estudiantes reflexivos, más que las de los otros estilos. En lo concerniente a las relaciones entre las variables predictoras, se encontró que a mayor planificación autorregulatoria mayor control y reflexión, como también a mayor control mayor reflexión. Esto pone de relieve el dinamismo e interacción entre las fases de autorregulación académica, concebidas desde la teoría de Pintrich (2000) como procesos vinculados y reiterativos. Por otra parte, se encontraron asociaciones moderadas entre las combinaciones de los estilos de aprendizaje activo-pragmático y reflexivo-teórico, así como relación entre el estilo pragmático con el reflexivo y el teórico. Estos hallazgos reafirman las combinaciones encontradas por los teóricos de los estilos de aprendizaje (Alonso et al., 1997), en el mismo orden de significación.

Aunado a lo anterior, se encontró que la fase de planificación autorregulatoria mostró una relación negativa con los estilos reflexivo y teórico; por las características de puntuación inversa de la escala de medición utilizada, a menor puntaje mayor autorregulación. Por su parte, la subescala control resultó estar asociada con el estilo de aprendizaje activo; estos resultados podrían sugerir que los estudiantes con preferencia por los estilos reflexivo y teórico presentan mayores niveles de planificación autorregulatoria, mientras que aquellos con preferencia por el estilo activo manifiestan mayores niveles de control. En lo que respecta al índice académico, se halló una asociación positiva con la modalidad de ingreso al sistema universitario por prueba interna y una correlación negativa con el ingreso por asignación de cupo. A partir de este importante resultado, puede inferirse que los estudiantes que logran su acceso a la universidad por la vía de aprobación de la prueba de ingreso presentan mayor relación con un rendimiento académico elevado; por el contrario, aquellos que ingresan a través de la asignación 
de un cupo se asocian con un rendimiento bajo. Este hallazgo revela cierto soporte y evidencia a los argumentos que se han expuesto, durante largo tiempo, en la discusión por la defensa en la implementación y aplicación de las pruebas de ingreso al sistema universitario, como una herramienta para seguir garantizando la excelencia académica del estudiantado.

En cuanto a la capacidad predictiva de la autorregulación, los estilos de aprendizaje y la modalidad de ingreso sobre el rendimiento académico se obtuvo que las variables que evidenciaron un peso significativo en la predicción del rendimiento, son: el control autorregulatorio, la preferencia por el estilo de aprendizaje teórico y el ingreso a la universidad en la modalidad de prueba interna. Estos hallazgos permitieron dar respuesta a las interrogantes inicialmente planteadas; asimismo, no se encontraron evidencias que puedan sugerir que la autorregulación, en sus fases de planificación y reflexión, así como algunos estilos de aprendizaje (reflexivo, pragmático, activo) y las modalidades de ingreso a la universidad vía CIU y asignación de cupos, tengan un papel significativo en la predicción del rendimiento académico.

Si bien los hallazgos reportados son relevantes desde un punto de vista teórico, se pretende que sean asumidos como una aproximación al estudio de la predicción del rendimiento académico que los alumnos de la Universidad Simón Bolívar alcanzan durante su primer curso de estudios superiores y que pueda replicarse el estudio en poblaciones académicas similares, con el propósito de verificar o discrepar de estos resultados. Para futuras investigaciones se sugiere incluir nuevos modelos teóricos y variables, realizando estudios transversales y/o longitudinales con estudiantes de educación media, para analizar su posible influencia en el comportamiento del aprendizaje autorregulado.

\section{Referencias}

Alonso, C., Gallego, D., \& Honey, P. (1997). Los estilos de aprendizaje: procedimientos de diagnóstico y mejora (3.a ed.). Madrid: Mensajero.
Álvarez, I. (2009). Evaluar para contribuir a la autorregulación del aprendizaje. Electronic Journal of Research in Educational Psychology, 7(3), 1007-1030.

Blumen, S., Rivero, C., \& Guerrero, D. (2011). Estilos de aprendizaje y rendimiento académico en estudiantes universitarios de educación a distancia. Revista Estilos de Aprendizaje, 7(7), 93-108.

Bolívar, J., \& Rojas, F. (2008). Los estilos de aprendizaje y el locus de control en estudiantes que inician estudios superiores y su vinculación con el rendimiento académico. Investigación y Postgrado, 23(3), 199-215.

Carbonero, M., \& Navarro, J. (2006). Entrenamiento de alumnos de educación superior en estrategias de aprendizaje en matemáticas. Psicothema, 18(3), 348-352.

Cardozo, A. (2008). Motivación, aprendizaje y rendimiento académico en estudiantes del primer año universitario. Laurus, 14(28), 209-237.

Cázares, A. (2009). El papel de la motivación intrínseca, los estilos de aprendizaje y estrategias metacognitivas en la búsqueda efectiva de información on line. Revista de Medios y Educación, 35, 73-85.

Cendrós, J., Aular, J., \& Marcano, N. (2008). Equidad para ingresar al sistema de educación superior: programa API-indígena de la Universidad del Zulia. Laurus, 14(28), 273-294.

Centro Interuniversitario de Desarrollo. (2006). Proyecto CINDA. Educación Superior en Iberoamérica 2006 (Informe de Venezuela). Caracas: Autor. Recuperado de http://www.carmengarciaguadilla.com/ articulos/2006_EducSup_en_Iberoamerica.pdf

Cerezo, M., Casanova, P, Torre, M., \& Carpio, M. (2011). Estilos educativos paternos y estrategias de aprendizaje en alumnos de educación secundaria. European Journal of Education and Psychology, 4(1), 51-61.

Craveri, A., \& Anido, M. (2009). El aprendizaje de matemática como herramienta computacional en el marco de la teoría de los estilos de aprendizaje. Revista Estilos de Aprendizaje, 3(3), 102-123.

De Garay, A. (2003). El perfil de los estudiantes de nuevo ingreso de las Universidades Tecnológicas en México. El Cotidiano, 19(122), 75-85.

De la Fuente, J., Pichardo, M., Justicia, F., \& Berbén, A. (2008). Enfoques de aprendizaje, autorregulación 
y rendimiento en tres universidades europeas. Psicothema, 20(4), 705-711.

De la Orden, A., Oliveros, L., Mafokozi, J., \& González, C. (2001). Modelos de investigación del bajo rendimiento. Revista Complutense de Educación, 12(1), 159-178.

De Miguel, M. (2001). Evaluación del rendimiento en la enseñanza superior: Resultados entre alumnos procedentes de la LOGSE y del COU. Oviedo, España: Centro de Investigación y Documentación Educativa.

Durán-Aponte, E., \& Pujol, L. (2012). Propiedades psicométricas del Cuestionario de Estilos de Aprendizaje (CHAEA) en estudiantes de carreras técnicas. Revista Educación y Futuro Digital, 3, 61-70.

Elvira-Valdés, M., \& Pujol, L. (2012). Autorregulación y rendimiento académico en la transición secundaria-universidad. Revista Latinoamericana de Ciencias Sociales, Niñez y Juventud, 10(1), 367-378.

Fuenmayor, L. (2002). A propósito de las iniquidades en el ingreso a la educación superior en Venezuela. Revista Venezolana de Gerencia, 7(17), 36-48.

Fuenmayor, L. (2003). El financiamiento universitario. Educere, 8(26), 385-395.

Garbanzo, G. (2007). Factores asociados al rendimiento académico en estudiantes universitarios: una reflexión desde la calidad de la educación superior pública. Educación, 31(1), 43-63.

García-Ros, R., \& Pérez-González, F. (2011). Validez predictiva e incremental de las habilidades de autorregulación sobre el éxito académico en la universidad. Revista de Psicodidáctica, 16(2), 231-250.

Gargallo, B. (2006). Estrategias de aprendizaje, rendimiento y otras variables relevantes en estudiantes universitarios. Revista de Psicología General y Aplicada, 59(1-2), 109-130.

Gargallo, B., Suárez, J., \& Ferreras, A. (2007). Estrategias de aprendizaje y rendimiento académico en estudiantes universitarios. Revista de Investigación Educativa, 25(2), 421-441.

González-Pienda, J., Núñez, J., Álvarez, L., \& González, S. (2002). Inducción parental a la autorregulación, autoconcepto y rendimiento académico. Psicothe$m a, 14(4), 853-860$.

Greene, J., Moos, D., Acevedo, R., \& Winters, F. (2008). Exploring differences between gifted and grade- level students use of self-regulatory learning processes with hypermedia. Computers $\mathcal{E}$ Education, 50(3), 1069-1083.

Gutiérrez, M., García, J., Vivas, M., Santizo, J., Alonso, C., \& Arranz, M. (2011). Estudio comparativo de los estilos de aprendizaje del alumnado que inicia sus estudios universitarios en distintas facultades de Venezuela, México y España. Revista Estilos de Aprendizaje, 7(7), 35-62.

Hair, J., Anderson, R., Tatham, R., \& Black, W. (2000). Análisis multivariante. Madrid: Prentice Hall.

Hernández, R., Fernández, C., \& Baptista, P. (2004). Metodología de la investigación. México: McGraw Hill.

Hervás, R. (2008). Identificación de variables que influyen en los estilos de aprendizaje: claves para conocer cómo aprenden los estudiantes. Revista Estilos de Aprendizaje, 1(1), 143-167.

Honey, P., \& Mumford, A. (1986). Using our learning styles. Berkshire, UK: Peter Honey.

Juárez, C., Hernández-Castro, S., \& Escoto, M. (2011). Rendimiento académico y estilos de aprendizaje en estudiantes de Psicología. Revista Estilos de Aprendizaje, 7(7), 77-92.

Keefe, J. (1982). Profiling and utilizing learning style. Reston, Virginia: NASSP.

Kerlinger, F., \& Lee, H. (2002). Investigación del comportamiento: métodos de investigación en ciencias sociales (4.a ed.). México: McGraw-Hill.

Lamas, H. (2008). Aprendizaje autorregulado, motivación y rendimiento académico. Liberabit. Revista de Psicología, 14, 15-20.

Laugero, L., Balcaza, G., Salinas, N., \& Craveri, A. (2009). Una indagación en el estilo de aprendizaje de los alumnos en distintos momentos de su vida universitaria. Revista Estilos de Aprendizaje, 4(4), 122-137.

Lezama, L. (2005). Perfil de orientación de metas, patrón de aprendizaje autorregulado y rendimiento académico (Tesis de maestría inédita). Universidad Simón Bolívar, Caracas.

Linares, Y. (2000). La educación superior: la reforma en diez planes del Ministerio de Educación, Cultura y Deportes. Educere, 4(10), 129-132. 
López-Aguado, M. (2011). Estilos de aprendizaje: diferencias por género, curso y titulación. Revista Estilos de Aprendizaje, 7(7), 109-134.

López, M., \& Silva, E. (2009). Estilos de aprendizaje: relación con motivación y estrategias. Revista Estilos de Aprendizaje, 4(4), 43-66.

Malmberg, J., Järvenoja, H., \& Järvelä, S. (2010). Tracing elementary school students' study tactic use in gStudy by examining a strategic and self-regulated learning. Computers in Human Behavior, 26(5), 1034-1042.

Marín, M. (2002). La investigación sobre diagnóstico de los estilos de aprendizaje en la enseñanza superior. Revista de Investigación Educativa, 20(2), 303-337.

Montero, E., Sepúlveda, M., \& Contreras, E. (2011). Estudio transversal de los estilos de aprendizaje y rendimiento académico en alumnos de ler. año de la carrera de Medicina Veterinaria. Revista Estilos de Aprendizaje, 7(7), 151-159.

Montes, J., Ayala, I., \& Atencio, D. (2005). Preparación para exámenes y aprendizaje autorregulado con estudiantes universitarios. Pensamiento Psicológico, 1(5), 57-71.

Morles, V., Medina, E., \& Álvarez, N. (2003). La educación superior en Venezuela (Informe). Caracas: IESALC-Unesco.

Núñez, J., Solano, P., González-Pienda, J., \& Rosário, P. (2006). El aprendizaje autorregulado como medio y meta de la educación. Papeles del Psicólogo, 27(3), 139-146.

Oficina de Planificación del Sector Universitario. (2007). Noticias Institucionales. Recuperado de http://www.opsu.gob.ve/extranet/rrpp/noticias

Oficina de Planificación del Sector Universitario. (2010). Pruebas internas de universidades no cuentan con respaldo del gobierno nacional. Recuperado de http://www.opsu.gob.ve/extranet/rrpp/noticias/ index.php?id_not $=329$

Parra, M. (2001). Educación superior en Venezuela: su perfil y perspectivas en un momento de transición. Tiempo de Educar, 3(6), 65-97.

Pérez, A., \& Díaz, M. (2006). La prueba de aptitud académica: una visión de la subprueba de comprensión de lectura. Investigación y Postgrado, 21(2), 143-176.

Pintrich, P. (2000). The role of goal orientation in selfregulated learning. En M. Boekaerts, P. Pintrich \&
M. Zeidner (Eds.), Handbook of self-regulation (pp. 451-502). San Diego: Academic Press.

Pujol, L. (2008). Búsqueda de información en hipermedios: efectos del estilo de aprendizaje y uso de estrategias metacognitivas. Investigación y Postgrado, 23(3), 45-67.

Rodríguez, S., Fita, E., \& Torrado, M. (2004). El rendimiento académico en la transición secundariauniversidad. Revista de Educación, 334, 391-414.

Segura, J. (2011). Tendencias de los estilos de aprendizaje de estudiantes y profesores en instituciones educativas venezolanas de bachillerato y formación técnica superior. Revista Estilos de Aprendizaje, 7(7), 160-183.

Suárez, J., \& Fernández, A. (2004). El aprendizaje autorregulado: variables estratégicas, motivacionales, evaluación e intervención. Madrid: UNED.

Schunk, D. (1998). Teorías del aprendizaje (2.a ed.). México: Pearson Educación.

Schunk, D. (2005). Commentary on self-regulation in school contexts. Learning and Instruction, 15(2), 173-177.

Universidad Simón Bolívar. (2007). Informe sobre el Sistema de Admisión de la USB. Caracas: Autor. Recuperado de http://sistema.cenda.usb.ve/gacetas/7286/ver

Universidad Simón Bolívar. (2010). Informe evaluación del Ciclo de Iniciación Universitaria-CIU. Caracas: Vicerrectorado Académico. Recuperado de http:// www.vracad.usb.ve/sites/default/files/documentos/ informe_ciu_05_09.

Valle, A., Canabach, R., Rodríguez, S., Núñez, J., \& González-Pienda, J. (2006). Metas académicas, estrategias cognitivas y estrategias de autorregulación del estudio. Psicothema, 18(2), 165-170.

Virtanen, P., \& Nevgi, A. (2010). Disciplinary and gender differences among higher education students in self-regulated learning strategies. Educational Psychology, 30(3), 323-347.

Zimmerman, B., Kitsantas, A., \& Campillo, M. (2005). Evaluación de la autoeficacia regulatoria: una perspectiva social cognitiva. Evaluar, 5(octubre), 1-21.

Zimmerman, B., \& Schunk, D. (2001). Self-regulated learning and academic achievement: Theoretical perspectives (2.a ed.). Nueva York: Springer-Verlag. 
